\title{
Acute Pericarditis in Crohn's Disease under Pharmacological Immunosuppression: A Diagnostic and Therapeutic Dilemma
}

\author{
Maria Trêpa, ${ }^{(1)}$ Isabel Neves, ${ }^{(0)}$ Marta Salgado, ${ }^{(1)}$ Graziela Carvalheiras, ${ }^{\left({ }^{\circ}\right.}$ Vasco Dias ${ }^{(1)}$ \\ Centro Hospitalar do Porto, Porto - Portugal
}

\section{Introduction}

Acute pericarditis in young patients usually has a benign course. However, in those with autoimmune diseases it is mandatory to consider the risk of opportunistic infections, and of association with the underlying disease or immunomodulatory drugs. This case report aims to show possible strategies for diagnosis and treatment of this particularly complex group of patients.

\section{Case Report}

A 40-year-old woman, with Crohn's disease diagnosed in 2009 was admitted to the emergency department with a 1-week history of fever, dyspnea and pleuritic chest pain. She was taking adalimumab (anti-TNF $\alpha$ ), and azathioprine was started after a negative screening for tuberculosis (TB) done by interferon gamma release assay (IGRA). Upon admission, examinations revealed pulmonary and peripheral congestion, and hypoxemia $\left(\mathrm{PaO}_{2}: 60 \mathrm{mmHg}\right)$. C-reactive protein was elevated (92 $\mathrm{mg} / \mathrm{L}$ ) and there was normocytic anemia (Hb: $7.9 \mathrm{mg} / \mathrm{dL}$ ) and lymphopenia (670/ $\mu \mathrm{L})$. Electrocardiogram showed $\mathrm{T}$ wave flattening and transthoracic echocardiogram (echo) showed moderate pericardial effusion and thickening of layers. Bi-ventricular function was normal.

A diagnosis of acute pericarditis complicated by effusion was assumed. Given the clinical context, she was admitted for further management. She underwent

\section{Keywords}

Crohn Disease; Pericarditis, Tuberculous; Fever; Tuberculosis; Anti-Bacterial Agents; AntibioticProphylaxis. exhaustive examination from which we inferred: (1) no criteria for myopericarditis; (2) no conditions for pericardial drainage (especially effusion on the posterior wall); (3) no microbiological isolates (i.e., bronchial lavage without Koch bacillus isolation); (4) undetermined interferon- $\gamma$ release assay (IGRA) in two different occasions; (5) negative serology for acute infections (HIV, CMV, EBV, HSV 1 and 2, toxoplasmosis, parvovirus); (6) positive immunological tests foranti-nuclear antibodies (ANA), anti-histones and immunoglobulins $\mathrm{G}$ and $\mathrm{M}$; (7) thoracic/abdominal/pelvic computed tomography angiography (Figure 1) with mediastinal and hilar adenopathies (not accessible) with no other findings.

The anti-TNF $\alpha$ was discontinued and she was started on ibuprofen and colchicine, showing an initially good clinical response and decrease of pericardial effusion. However, reassessment 15 days after discharge showed an increase in pericardial effusion and echo criteria for constrictive pericarditis: septal bounce, increased respiratory variability ( $40 \%$ ) of the transtricuspid flow, and dilatation and reduced variability of the inferior vena cava (Figure 2).

Given the unfavorable course, four possible etiologies of pericarditis were considered: (1) idiopathic/viral, (2) bacterial (especially TB), (3) extra-intestinal manifestation of inflammatory bowel disease (IBD) and (4) association with immunomodulation therapy.

The case was presented in a multidisciplinary meeting (including Cardiology, Internal Medicine and Immunology specialists) that concluded that there were no definitive criteria for the cause of pericarditis. However, because of the evidence suggestive of TB (immunosuppression, mediastinal adenopathy and incipient constrictive pericarditis), the real possibility of an adverse outcome if TB was not treated, as well as an increased risk of worsening with the use of corticosteroid

Mailing Address: Maria Trêpa

Largo Prof. Abel Salazar, n 1. Postal Code: 4099-001, Porto - Portugal.

E-mail: maria_trp@hotmail.com 


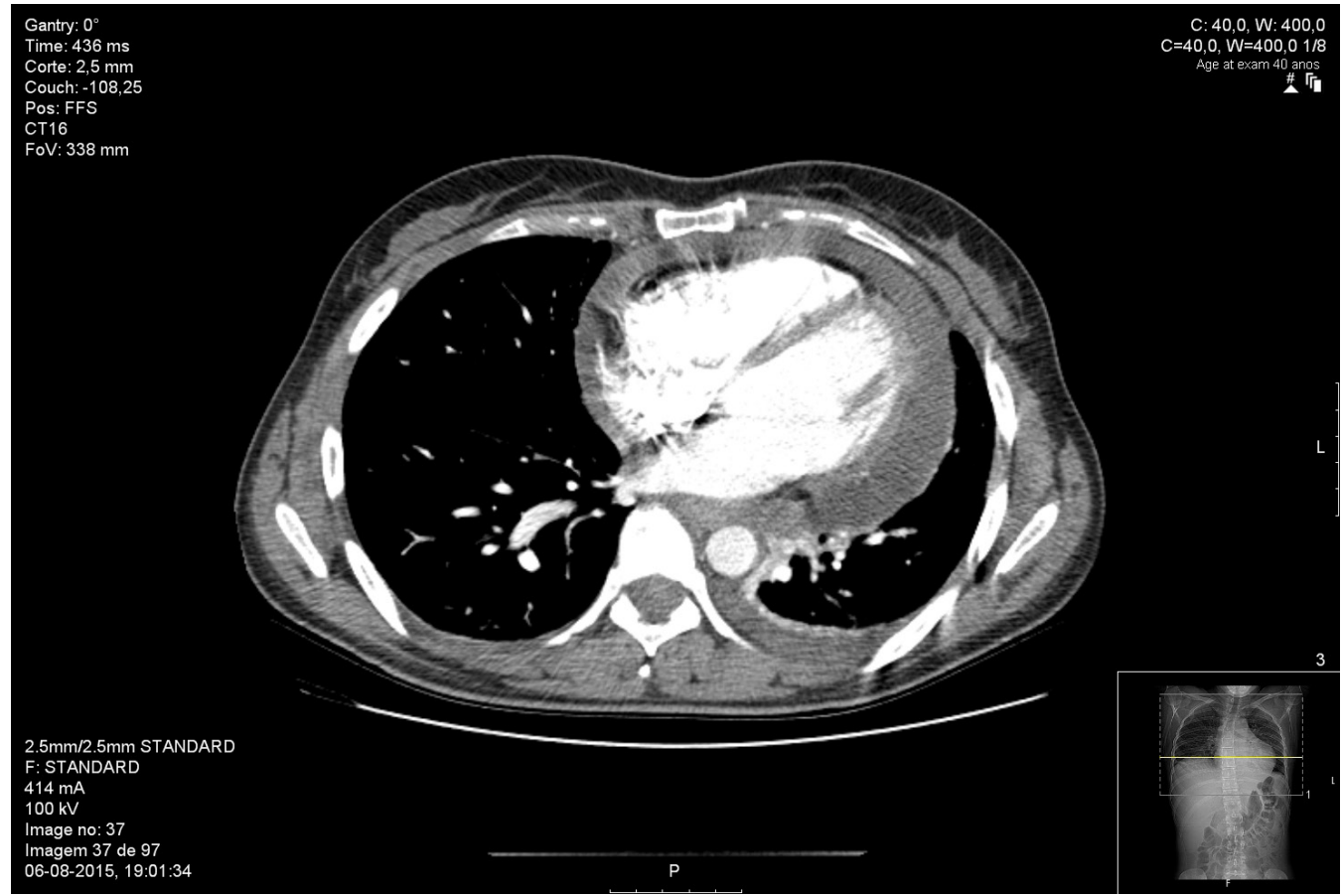

Figure 1 - Thoracic computed tomography angiography showing moderate pericardial effusion with asymmetric distribution.

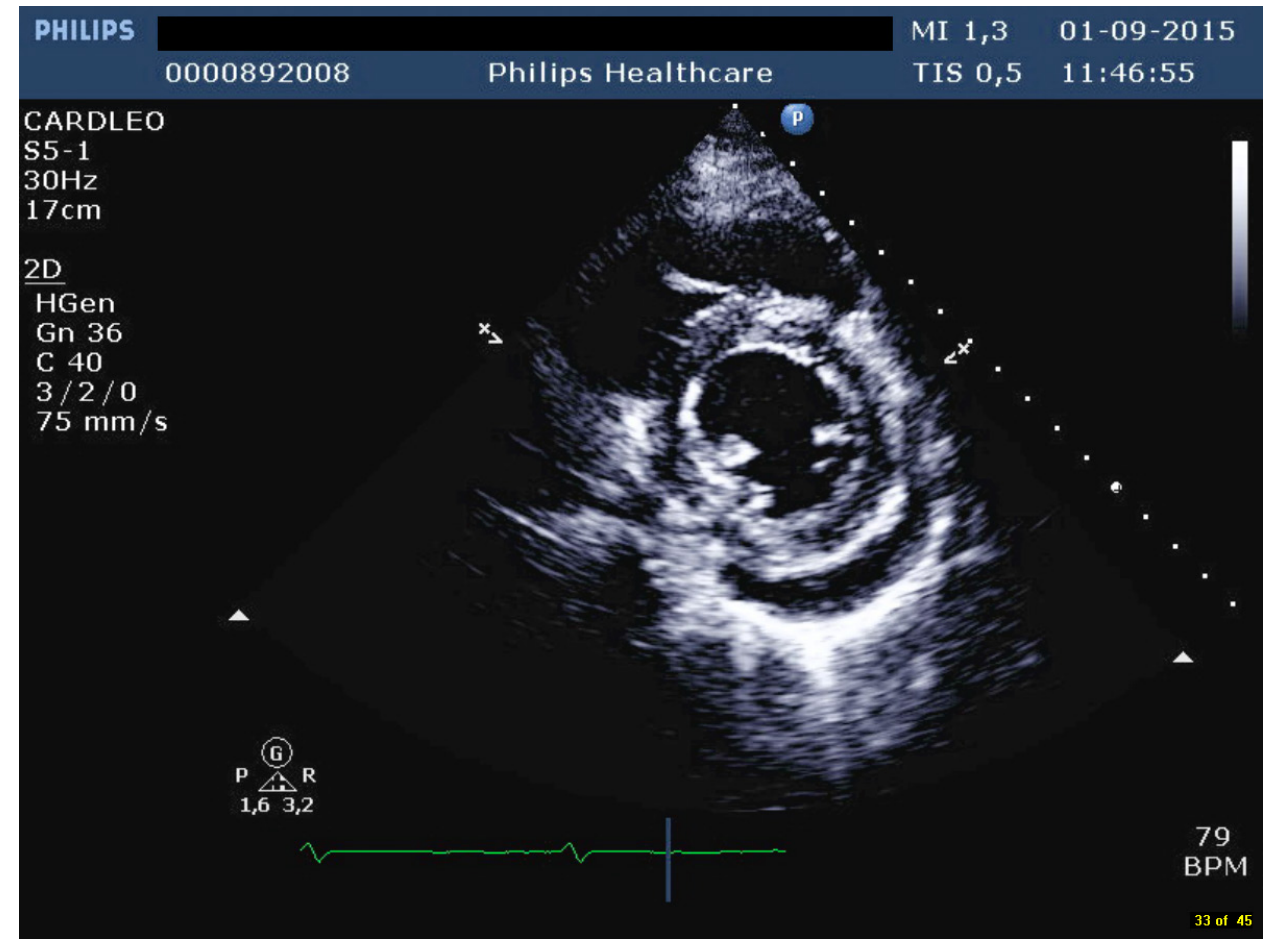

Figure 2 - Control echocardiogram performed two weeks after treatment initiation showing thickening of pericardial layers and moderate effusion, septal bounce and increased respiratory variability of the transtricuspid flow. 
alone, we decided to assume the presumptive diagnostis of tuberculous pericarditis. We maintained the AntiTNF $\alpha$ discontinued and started corticosteroids and anti-TB drugs (isoniazid and rifampicin for 6 months and pyrazinamide and ethambutol for 2 months).

The patient showed progressive improvement. Control echocardiography at one and eight months after ending TB-treatment revealed complete resolution of effusion and of constriction criteria.

\section{Discussion}

As the number of immunosuppressed patients grows, the diagnostic and therapeutic dilemma reported here will probably increase since these patients are particularly complex to manage. Viral etiology of pericarditis remains the most frequent one, but it is mandatory to consider not only the association with the underlying autoimmune disease or with immunomodulation therapy but also the risk of opportunistic infections.

Pericarditis was first described as an extra-intestinal manifestation of irritable bowel disease (IBD) in $1967 .{ }^{1}$ It's the most frequently reported cardiac complication and has various presentations, since self-limited forms until severe perimyocarditis and cardiac tamponade, ${ }^{2-6}$ The most accepted pathophysiological mechanism is based on the pericardial aggression due to systemic inflammation. An Italian study ${ }^{3}$ reported a possible relationship between pericardial effusion and IBD flares. The development of pericarditis does not seem to be related to disease chronicity and has been described both independently and in association with IBD flares. ${ }^{5}$

There are also reports of pericarditis as a complication of immunomodulatory therapy itself, such as associated with 5-ASA, azathioprine and anti-TNF $\alpha$ drugs. Accepted explanations include lupus-like reactions, type 3 hypersensitivity and pro-inflammatory effect of some drugs in serous membranes..$^{6-8}$ Usually, there is a clear temporal relation with the initiation of drug use, with improvement after its discontinuation and an increase in anti-histone antibodies. In our case, only the antibody was elevated making it hard to establish a causal relationship. The approach would be to discontinue the offending drug and initiate corticosteroid therapy.

The presumptive diagnosis of TB was assumed after a multidisciplinary meeting and careful consideration. $\mathrm{TB}$ prevalence is still high in the urban region where our patient lives. There is a known correlation between anti-TNF $\alpha$ and TB reactivation that justifies mandatory screening before starting treatment. In case of $\mathrm{TB}$ pericarditis, a definitive diagnosis is notoriously difficult due to the low yield of cultures and biopsies, and usually a presumptive or exclusion diagnosis is made. The progression to constrictive pericarditis is a potentially serious complication. Without a targeted therapy, up to $50 \%$ of patients with effusive-constrictive physiology might progress to constriction in six months. ${ }^{9}$ Several studies reinforce the need to consider $\mathrm{TB}$ pericarditis in patients at increased risk for TB development with nonbenign evolution. In the absence of Koch bacillus isolation from the pericardial fluid or tissue, TB pericarditis is considered likely if the bacillus is isolated from sources and/or if there is a favorable response (based on symptoms and echo) to anti TB-drugs. ${ }^{10}$ In patients taking anti-TNF $\alpha$ drugs, there is a particularly increased risk of $\mathrm{TB}$, frequently extra-pulmonary.

The impossibility of performing pericardiocentesis was a limiting factor in our diagnostic approach. However, in the absence of other strong diagnostic hypotheses, and given the treatment with anti-TNF $\alpha$, the high TB prevalence, the angioCT and echo findings, and the possibility of irreversible adverse outcome if untreated, we assumed the presumptive diagnosis of TB pericarditis. The good response to treatment favored our decision.

This case report portraits the difficulties in the diagnosis of these patients, but also provides possible therapeutic strategies. In the absence of strong recommendations for such complex cases, we trust in experience and multidisciplinary discussion. These cases should be managed with specific strategies defined individually.

\section{Author contributions}

Conception and design of the research: Trêpa M, Neves I, Dias V. Acquisition of data: Trêpa M, Neves I, Dias V. Analysis and interpretation of the data: Trêpa M, Salgado M, Carvalheiras G. Writing of the manuscript: Trêpa M. Critical revision of the manuscript for intellectual content: Trêpa M, Neves I, Salgado M, Carvalheiras G, Dias V.

\section{Potential Conflict of Interest}

No potential conflict of interest relevant to this article was reported.

\section{Sources of Funding}

There were no external funding sources for this study. 


\section{Study Association}

This study is not associated with any thesis or dissertation work.

\section{References}

1. Young PC. Colonic and systemic manifestations of chronic ulcerative colitis. Med Clin North Am. 1967;51(4):1011-3.

2. Bernstein $\mathrm{CN}$, Blanchard JF. The clustering of other chronic inflammatory diseases in inflammatory bowel disease: a population-based study. Gastroenterology.2005;129(3):827-36

3. Bragagni G, Brogna R, Franceschetti P, Zoli G.et al. Cardiac involvement in Crohn's disease: echocardiographic study. J Gastroenterol Hepatol. 2007. 22(1):18-22.

4. Patwardhan RV, Heilpern RJ, Brewster AC, Darrah JJ. Pleuropericarditis: an extraintestinal complication of inflammatory bowel disease. Report of three cases and review of literature. Arch Intern Med. 1983;143(1):94-6.

5. Stasinopoulou P, Kaziani A, mantzaris G, Roussos A, Skoutelis A. Parallel manifestation of Crohn's disease and acute pericarditis: a report of two cases. Int J Colorectal Dis. 2007;22(9):1123-5.

\section{Ethics approval and consent to participate}

This article does not contain any studies with human participants or animals performed by any of the authors.

6. Kiyomatsu H, Kawai K, Tanaka T, Tanaka J, Kiyomatsu T, Nozawa H, et al. Mesalazine-induced pleuropericarditis in a patient with Crohn's disease. Intern Med. 2015;54(13):1605-8.

7. Burke JP, Kelleher B, Ramadan S, Quinlan M, Sugrue D, O'Donovan MA, et al. Pericarditis as a complication of infliximab therapy in Crohn's disease. Inflamm Bowel Dis. 2008;14(3):428-9.

8. Devasahayam J, Pillai U. A rare case of pericarditis, complication of infliximab treatment for Crohn's disease. J Crohns Colitis. 2012;6(6):730-1.

9. Adler Y, Charron F, Imazio M, Badano L, Barón-Esquivias G, Bogaert J. 2015 ESC Guidelines for the Diagnosis and Management of Pericardial Diseases. Rev Esp Cardiol. (Engl Ed). 2015;68(12):1126.

10. Stout J, von Reyn FC, Baron EL. Tuberculous pericarditis. [internet]. [Cited in 2017 May 12]. Available from: https://www.uptodate.com/ contents/tuberclous-pericarditis 